\title{
Outstanding Methane Oxidation Performance of Pd-Embedded Ceria Catalysts Prepared by a One-step Dry Ball-Milling Method
}

\author{
Maila Danielis,[a] Sara Colussi,[a] Carla de Leitenburg,[a] Lluís Soler,[b] Jordi Llorca,[b] and \\ Alessandro Trovarelli*[a]
}

[a] M. Danielis, dr. S. Colussi, prof. C. de Leitenburg, prof. A. Trovarelli

Polytechnic Department. University of Udine. Via del Cotonificio 108, 33100 Udine, Italy

[b] dr. L. Soler, prof. J. Llorca

Institute of Energy Technologies, Department of Chemical Engineering and Barcelona Research Centre in Multiscale Science and Engineering. Universitat Politècnica de Catalunya, EEBE. Eduard Maristany 10-14, 08019 Barcelona, Spain

\begin{abstract}
By carefully mixing Pd metal nanoparticles with $\mathrm{CeO}_{2}$ polycrystalline powder under dry conditions a new unpredicted arrangement of the $\mathrm{Pd}-\mathrm{O}-\mathrm{Ce}$ interface is obtained, where an amorphous shell containing Palladium species dissolved in Ceria is covering a core of $\mathrm{CeO}_{2}$ particles. The robust contact that is generated at nanoscale, along with mechanical forces generated during mixing, promotes the redox exchange between $\mathrm{Pd}$ and $\mathrm{CeO}_{2}$ and creates highly reactive and stable sites constituted by $\mathrm{PdO}_{x}$ embedded into $\mathrm{CeO}_{2}$ surface layers. This specific arrangement outperforms conventional $\mathrm{Pd} / \mathrm{CeO}_{2}$ reference catalysts in methane oxidation, by lowering light-off temperature by more than 50 degrees and boosting reaction rate. The origin of the outstanding activity is traced back to the structural properties of the interface, modified at nanoscale by mechano-chemical interaction, and it is unraveled by a combined set of experimental data including high resolution transmission electron microscopy and supported by recent computational studies.
\end{abstract}

The increasing concern over the abundant emissions of greenhouse gases from motor vehicles is pushing towards the development of more efficient catalysts for their abatement. This is particularly true for natural gas fueled vehicles, for which the exponential growth of the market and the concern for methane global warming potential urge for the design of catalytic systems with improved activity at low temperature and higher resistance to deactivation under operating conditions.[1] An efficient low temperature activation of the $\mathrm{CH} 4$ molecule would also be a significant advancement in the field of methane utilization, an issue that is now attracting several efforts due to the increased supplies of shale gas and the consequent availability of natural gas as a feedstock.[2] Pd-based formulations are the most effective for methane oxidation, and the use of ceria as support confers additional benefits to the catalysts due to its unique redox features and to the level of Pd-Ce interfacial interactions.[3] It is reported from experimental[3b, 4] and computational[5] studies that an enhanced Pd-ceria interaction can improve significantly the catalytic activity of these materials. In particular, the presence of $\mathrm{Pd}$ into ceria lattice can lead to the formation of highly reactive $\mathrm{Pd} 2+/ 4+$ sites which show lower methane activation barriers compared to isolated PdOx units. Interestingly, differently from what happens on PdOx clusters, on these ionic Pd species methane activation proceeds via hydrogen abstraction, a route that is potentially very important for methane utilization.[5a]

We have already reported the increased activity of a $\mathrm{Pd} / \mathrm{CeO} 2$ catalyst prepared by solution combustion synthesis in which the substitution of $\mathrm{Pd} 2+$ ions into ceria lattice caused the formation of ordered arrays of oxygen vacancies and highly reactive undercoordinated oxygen atoms.[6] More recently we also investigated the milling of CeO2-based materials with carbon, originating a 2D carbon layer, covering the ceria particles, and improving the interfacial redox 
exchange between the two materials.[7] Here, by combining the above mentioned approaches, we use a controlled one-step dry milling procedure where Pd metal nanoparticles are put in contact with ceria particles to prepare a methane oxidation catalyst that outperforms traditional $\mathrm{Pd} / \mathrm{CeO} 2$ due to the unique structural arrangements that characterize metal/support interface at nanoscale. In addition, avoiding the use of Pd nitrate or chloride solution significantly reduces waste generation ensuring lower environmental impact. The characteristics of the preparation method, the properties and performances of the catalyst have been investigated in detail; the data suggest a correlation between the unusual morphology developed at nanoscale and the high catalytic activity observed, and this correlation is supported by most recent theoretical simulations.[5]

The mechanically mixed samples (denoted with $\mathrm{M}$ ) were prepared by mixing together metallic $\mathrm{Pd}$ nanoparticles with $\mathrm{CeO} 2$ powder aged at $1173 \mathrm{~K}$ in a mini ball mill, to obtain a nominal $\mathrm{Pd}$ loading of $1 \% \mathrm{wt}$. (PdCeM). Reference catalysts with the same nominal Pd loading were also synthesized by incipient wetness impregnation on the same $\mathrm{CeO} 2$ support (PdCelW) and by solution combustion synthesis (PdCeSCS). An additional catalyst, where PdO nanoparticles were used in substitution of $\mathrm{Pd}$ metal in the milling procedure, was also prepared for comparison (PdOCeM). To check the effect of the support, comparison of PdCeM with Pd supported on ZrO2 (PdZrM) prepared by the same procedure was also carried out. The details on samples preparation and the milling parameters are reported in the Supporting Information, along with the detailed description of testing conditions and characterization methods. Stability under reaction conditions and durability were tested in comparison to PdCeIW following six reaction cycles up to $1173 \mathrm{~K}$ and monitoring time on stream behavior also under hydrothermal conditions. Comparison of X-ray diffraction and temperature programmed reduction profiles of PdCeM, PdCeIW and PdOCeM are reported in Figure S1 and S2. The light-off curves of methane combustion under lean atmosphere for the different Pdceria formulations are shown in Figure 1, along with the corresponding cooling part of the cycle.

In these experiments the catalysts were cycled under reaction conditions between room temperature and $1173 \mathrm{~K}$ for 2 heating/cooling cycles and the second cycle was selected as representative of the catalytic behavior, unless otherwise stated. Interestingly, the overall performance of the $\mathrm{M}$ samples strongly depends on the nature of the palladium precursor. $\mathrm{PdCeM}$ has a much higher activity than PdOCeM in the whole temperature range, and its behavior outperforms significantly that of the impregnated sample and of the catalyst made by solution combustion synthesis, which was reported as one of the best literature examples.[3b, 6] This can be seen either by the lower light-off temperature or by the higher reaction rate measured for the PdCeM sample in a recycle reactor, as reported in Table 1. 


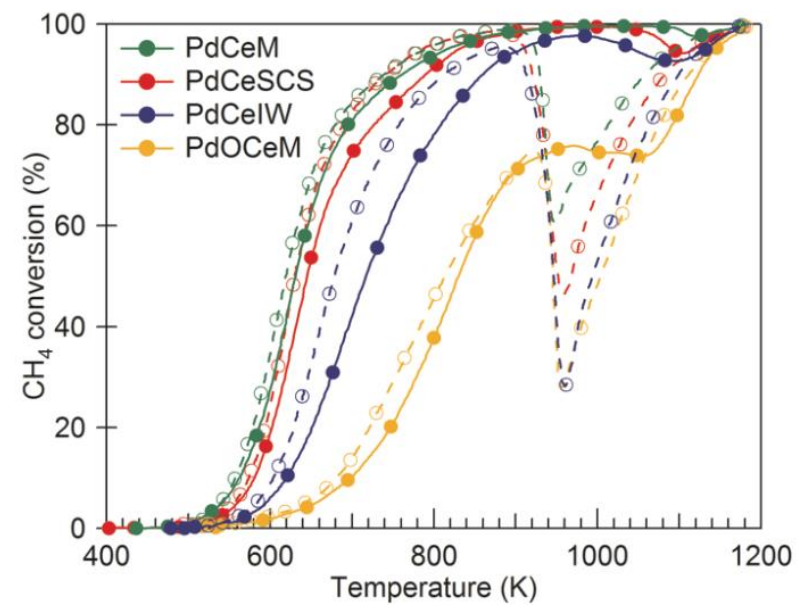

Figure 1. Light-off curves for Pd-CeO2 catalysts (2nd cycle). Solid line, closed symbols: heating part of the cycle; dashed line, open symbols: cooling part of the cycle. Conditions: GHSV ca. $200000 \mathrm{~h}-1,0.5 \% \mathrm{CH} 4,2 \% \mathrm{O} 2$, He to balance.

Table 1. Physico-chemical properties and activity parameters for methane combustion.

Table 1. Physico-chemical properties and activity parameters for methane combustion.

\begin{tabular}{lcccc}
\hline Sample & $\begin{array}{c}\text { Pd loading } \\
(\mathrm{wt} \%)^{[\mathrm{a}]}\end{array}$ & $\begin{array}{c}\text { Surface area } \\
\left(\mathrm{m}^{2} / \mathrm{g}\right)\end{array}$ & $\begin{array}{c}\mathrm{T1}{ }^{[\mathrm{b}]} \\
(\mathrm{K})\end{array}$ & $\begin{array}{c}\text { Reaction rate }^{[\mathrm{cc}]} \\
\left(\mu \mathrm{mol} / \mathrm{g}_{\mathrm{Pd}}{ }^{*} \mathrm{~S}\right)\end{array}$ \\
\hline PdCeM & 0.81 & 3.2 & 564 & 208 \\
PdOCeM & 0.80 & 4.1 & 700 & 16 \\
PdCeIW & 0.97 & 2.3 & 619 & 32 \\
PdCeSCS & 0.93 & 5.9 & 581 & 112 \\
\hline
\end{tabular}

[a] measured by ICP elemental analysis; [b] temperature for $10 \%$ conversion; [c] measured at $623 \mathrm{~K}$ in a recycle reactor.

The better performance of PdCeM is maintained also in the cooling portion of the cycle, where the drop of activity due to the dynamics of Pd-PdO transformation follows the order $P d C e M<P d C e S C S<P d O C e M<P d C e I W$. The catalyst stability has been successfully checked over six light-off cycles (Figure S3) where it can be seen that PdCeM shows a stable $\mathrm{CH} 4$ conversion behavior from the third cycle onward. Time on stream behavior has also been investigated both under reaction conditions and in the presence of large excess of water (Figure S4), which is known to accelerate deactivation in Pd-based catalysts. [8] Catalysts prepared by milling are more stable with an overall activity loss of ca. $25 \%$ after $24 \mathrm{~h}$ on stream, compared with a loss of $70 \%$ observed in reference PdCelW.

Light-off activity behavior of PdCeM is also affected by the modification of milling parameters. It is observed that, increasing milling intensity, the overall light-off profiles of the catalyst shift to higher temperatures, indicating a drop of reaction rates compared to our standard PdCeM sample (see Figure S5). 
Also, milling under loose conditions without using balls does provide catalysts active in a first cycle at low temperature but suffering of dramatic deactivation, due to the lack of nanoscale interactions between Pd and ceria and extensive sintering at higher temperatures (Figure S6). This shows that the choice of our milling parameters for PdCeM sample (10 minutes milling at an oscillation frequency of $15 \mathrm{~Hz}$ with a ball to powder ratio of 10) optimizes activity performances.

Methane oxidation light-off profiles were also collected on the corresponding $\mathrm{ZrO} 2$ supported catalysts, and they did not evidence any significant difference among samples prepared by impregnation and by mechanical milling (Figure S7). This strongly supports the fact that the origin of the unique activity of $\mathrm{PdCeM}$ must be found in a specific characteristic of the $\mathrm{Pd}-\mathrm{CeO} 2$ interface that is promoted during milling, and not in a more general behavior of samples prepared by a mechanochemical procedure.

In parallel to light-off cycles, temperature programmed oxidation experiments (TPO, Figure 2) showed for fresh PdCeM the oxidation of Pd at low temperature (cycle 1), and the presence of at least three $\mathrm{PdO}$ decomposition peaks (cycles 2 and 3 ), indicating the coexistence of different palladium species. Quantitative re-oxidation of Pd during cooling occurs in one single peak at ca. $910 \mathrm{~K}$. A comparison with PdCelW, which presents only two oxygen release peaks, is shown in Figure $\mathrm{S} 8$.

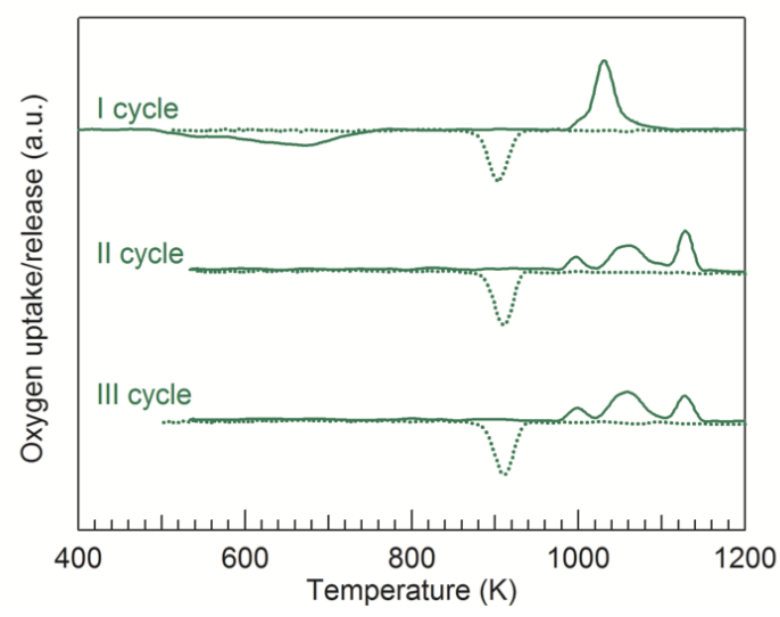

Figure 2. TPO profiles for PdCeM over three consecutive heating/cooling cycles. Solid line: heating ramp; dotted line: cooling ramp.

Details on the $\mathrm{Pd}-\mathrm{CeO} 2$ morphology were obtained by HRTEM analysis on the fresh samples. Figure $3(A, B)$ shows the surface of PdCelW where, as expected, small Pd nanoparticles (ca. 2 $\mathrm{nm}$ ) are well dispersed over a clean ceria surface. In contrast, PdCeM is characterized by ceria crystallites that are covered by an amorphous layer measuring between 2 and $5 \mathrm{~nm}$ in thickness(Figure $3 \mathrm{C}-\mathrm{F}$ ). This amorphous shell is compact and perfectly defined, following the perimeter of the ceria particles (Figure 3C-D). Interestingly, the ceria crystallites covered by this layer present a more rounded morphology, suggesting that the spreading of $\mathrm{Pd}$ by mechanical mixing affects their surface. Ceria nanoparticles show well-defined lattice fringes at 3.12 and $2.71 \AA$ in both samples, which correspond to the (111) and (200) crystallographic 
planes of $\mathrm{CeO} 2$, respectively. In Figure 3D the EDX analysis of the shell is reported, which contains both ceria and $\mathrm{Pd}$ (the $\mathrm{Cu}$ signal originates from the TEM grid), indicating that the shell is comprised of a mixed Pd-Ce phase.

In addition to this amorphous layer, some smaller particles measuring less than $5 \mathrm{~nm}$ are also detected, mostly decorating the ceria crystallites (Figure $3 \mathrm{E}, \mathrm{F}$ ). Lattice fringe analysis of these nanoparticles (see insets " $c$ " and " $d$ " corresponding to FT images of "a" and " $b$ ", respectively, in Figure 3F) shows fringes at 2.25 and $1.95 \AA$. They correspond to the (111) and (200) crystallographic planes of Pd metal. Noticeably, these Pd nanoparticles are embedded in the amorphous shell, which suggests that the shell is produced by the distribution of Pd metal nanoparticles over the ceria support upon mechanical mixing. Figure $3 \mathrm{E}$ represents nicely this situation: a very small nanoparticle (less than $2 \mathrm{~nm}$ ) diffuses into the shell at the edges, still preserving some crystallographic order (see the FT image in inset "a" showing fringes of Pd metal).

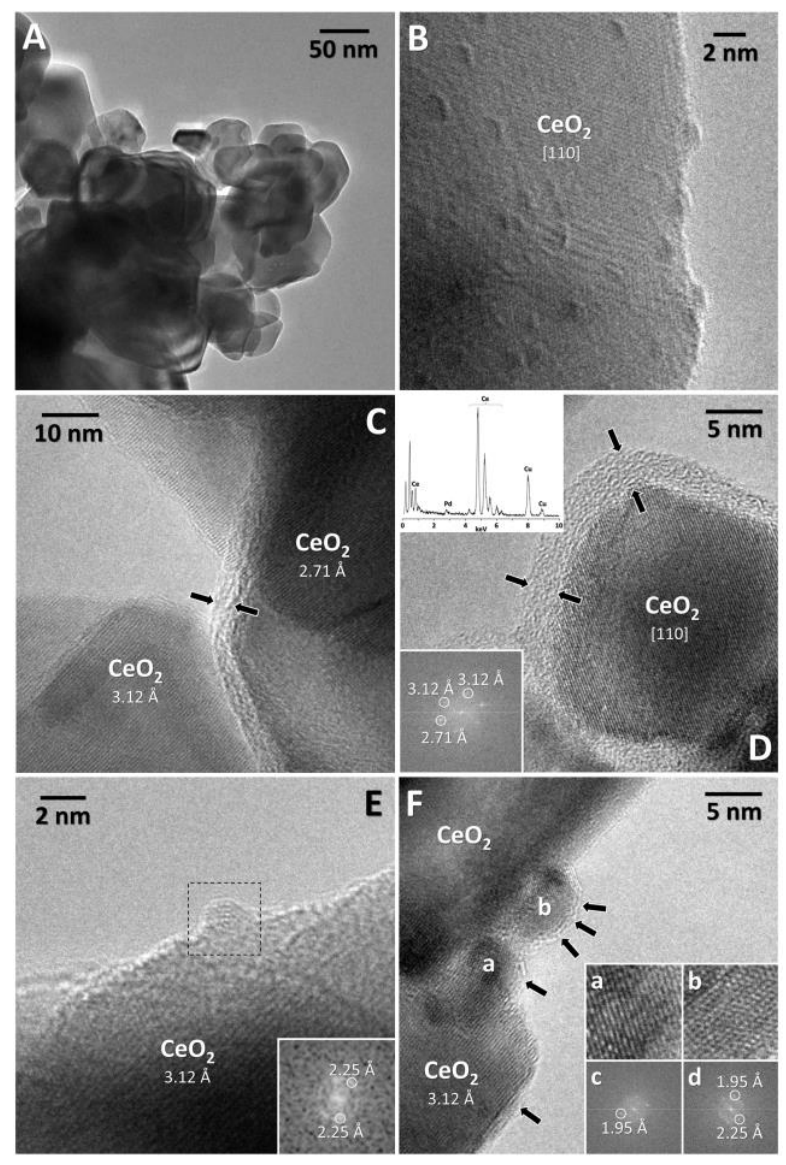

Figure 3. HRTEM images of PdCeIW (A,B) and PdCeM (C-F).

Remarkably, this morphology is not observed on PdOCeM and PdZrM where well defined PdO and Pd nanoparticles are respectively found over the support (see Figure S9 and S10 for representative samples). Moreover, the mechanical milling of pure $\mathrm{CeO} 2$ under the same conditions does not induce any change of the surface (Figure S11) indicating that surface amorphisation takes place only upon mixing of $\mathrm{Pd}$ and ceria. 
A similar core-shell structure where $\mathrm{Pd}$ covers $\mathrm{SnO} 2$ particles was observed on a $\mathrm{Pd} / \mathrm{SnO} 2$ catalyst prepared by wet impregnation;[9] however, the Pd-Sn alloy based shell disappeared under oxidizing atmosphere. Conversely, the amorphous shell observed on PdCeM is also stable under reaction conditions, as demonstrated by the HRTEM image of the used sample after two cycles (Figure $4 \mathrm{~A}$ and $\mathrm{B}$ ) in which it is possible to recognize the presence of two fringes, one at $3.1 \AA$ corresponding to the (111) crystallographic planes of the ceria substrate, and another one at a greater spacing likely due to inclusion of PdO within ceria lattice, where $\mathrm{Pd}$ is strongly interacting with the support. This is in accordance with the existence of small domains of Pd completely embedded in the amorphous shell, as discussed above for the fresh PdCeM sample. Figure 4 ( $C$ and $D$ ) shows representative images of the shell around ceria after 6 reaction cycles, which is marked between arrows along with the crystallographic planes exposed by the ceria crystallites. From these images it is clear that the shell is preserved after the sixth cycle. Interestingly, no remaining Pd containing particles have been identified indicating that all Pd is still spread within the layer surrounding the ceria particles. Similarly, time on stream behavior under hydrothermal conditions does not alter the structure of the layer, indicating stability also in the presence of excess of water (Figure S12).

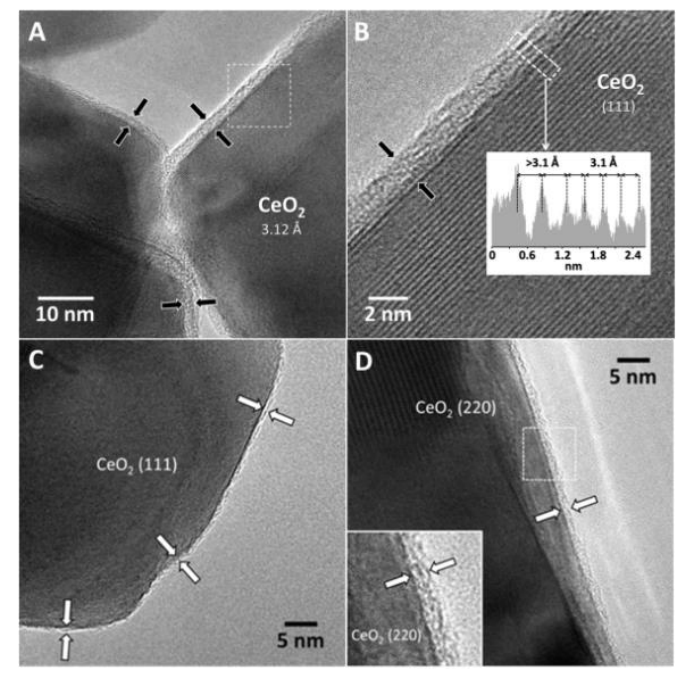

Figure 4. HRTEM images of PdCeM after two $(A, B)$ and six $(C, D)$ methane oxidation tests.

The reasons behind the structural rearrangement of the surface in PdCeM during milling are not straightforward and should be the result of a combination of different elements. Some interesting indications on the phenomena that might take place during the mechanical mixing of $\mathrm{Pd}$ and ceria powders come from the studies on solid state amorphisation.[10] In these studies it is evidenced that mechanical alloying increases vacancies, chemical disordering at atomic level and nanocrystalline grain boundaries, which in turn raise the free energy of the crystalline phase favoring the stabilization of the amorphous state, particularly at nanometer scale. This process usually starts with the amorphisation of the edges, and in some cases proceeds through the formation of a crystalline solid solution which is then transformed into the amorphous phase. Interestingly, the formation of point and lattice defects by milling has been proposed as the rate limiting step for amorphisation.[11] 
It should be observed though that, in general, in the studies dealing with solid state amorphisation the energies employed are much higher than those of our dry mixing method, nevertheless in the case of palladium and ceria the process might be speeded up by other factors, like the specific interaction between Pd and CeO2.[12] This interaction, which could account for an easier solid state reaction between the two components, involves the formation of vacancies and/or more active oxygen states at the metal-oxide interface as observed for other systems after milling.[13] In addition, it has been reported that the oxidation state of cerium ions in solid solutions can be altered at room temperature by changing mechanically the local stress state, and that the distribution of $\mathrm{Ce} 3+/ \mathrm{Ce} 4+$ can be influenced by stress gradients.[14]

Indeed, XPS studies carried out on PdCeM and PdCeIW after one reaction cycle (Table 2) show that the ratio between $\mathrm{Ce}(\mathrm{III})$ and $\mathrm{Ce}(\mathrm{IV})$ is higher on the sample prepared by mechanical grinding compared to the impregnated one, and also to pure milled $\mathrm{CeO} 2$ (sample CeM). This indicates that the interplay between $\mathrm{Pd}$ and ceria on $\mathrm{PdCeM}$ is stronger and induces a higher degree of ceria reduction. In addition, a better and more homogeneous Pd distribution is also observed in milled samples which is maintained after the first reaction cycles.

Table 2. Data from XPS analysis.

\begin{tabular}{lccccc}
\hline Sample & $\mathrm{Pd} / \mathrm{Ce}$ & $\mathrm{Ce}(\mathrm{III}) / \mathrm{Ce}$ & $\mathrm{Pd}(0)$ & $\mathrm{Pd}(\mathrm{II})(\%)$ & $\mathrm{Pd}(\mathrm{IV})(\%)$ \\
\hline CeM & - & 0.16 & - & - & - \\
PdCeM $^{\mathrm{a}}$ & $0.19(0.18)^{\mathrm{b}}$ & $0.24(0.12)$ & $0(0)$ & $82(78)$ & $18(22)$ \\
PdCeIW $^{\mathrm{a}}$ & $0.15(0.15)$ & $0.15(0.12)$ & $0(0)$ & $76(87)$ & $24(13)$ \\
\hline
\end{tabular}

[a] collected after one reaction cycle; [b] in parentheses data collected after six reaction cycles.

Characterization after six reaction cycles shows an increase of $\mathrm{Pd}(\mathrm{IV})$ in milled sample compared to PdCelW while the amount of $\mathrm{Ce}$ (III) become similar, likely due to prolonged oxidation cycles at high temperature. In addition, the better Pd distribution of PdCeM is maintained after stability tests. This is also confirmed by comparing the Raman spectra obtained by mapping 144 ?m2 of the surface of PdCeM and PdCelW (Figure S13). In both samples the more intense signal is due to the $\mathrm{F} 2 \mathrm{~g}$ vibrational mode of $\mathrm{CeO} 2(461 \mathrm{~cm}-1)$, but a signal originating from the $\mathrm{B} 1 \mathrm{~g}$ vibrational mode of $\mathrm{PdO}$ is also observed $(646 \mathrm{~cm}-1)$. Collecting several spectra, it is clearly seen that the intensity ratio between the two signals is rather constant for PdCeM (Figure S13), indicating a more homogeneous spreading of Pd over $\mathrm{CeO} 2$, as it can be expected from its distribution within the surface layer. A broad Raman signal covering the region between $550-600 \mathrm{~cm}-1$ is also detected in PdCeM; peaks in this region are attributed to surface defects in ceria[15] that might include those generated by close interaction and inclusion of $\mathrm{Pd}$ into $\mathrm{CeO} 2$ lattice.

The information gathered from HRTEM and XPS studies show that on PdCeM there is a unique arrangement of $\mathrm{Pd}$ and ceria that is not observed on samples prepared by conventional routes. This arrangement is the result of an interaction between $\mathrm{Pd}$ and ceria promoted by mechanical milling and by the redox characteristics of the two components. We recently reported that the milling of ceria with carbon soot results in the formation of a $\mathrm{CeO} 2$ core wrapped in a soft 
carbon shell[7]; the nanoscale arrangement created by mixing was promoted by the different hardness of the two materials that helps the spreading of the softer carbon particles on the surface of ceria. This might explain why the core-shell structure observed here is obtained only when mixing Pd metal nanoparticles and ceria and not when harder materials like PdO and/or ZrO2 are used (0.6 vs 4 GPa for Pd metal and ceria, respectively, against 8 for zirconia[16]). It is true in fact that the adhesive force between two compressed particles varies inversely with their hardness.[17]

The high reactivity for methane oxidation over $\mathrm{Pd} / \mathrm{CeO} 2$ interfacial sites was recently described by the group of Janik, using integrated DFT and empirical reactive force field methods[5b]. In their study partially embedded Pd clusters are shown to favor the formation of metastable transient $\mathrm{Pd} 4+$ ions inserted into ceria lattice which are indicated as the most reactive for methane activation. The authors predict that, starting from supported Pd clusters, Pd atoms can migrate across the ceria surface and assume a higher oxygen coordination being incorporated into the lattice when encountering steps, vacancies and/or grain boundaries, forming active oxidized Pd species. It is remarkable to observe that this description well agrees with our HRTEM observation of nanometer scale Pd clusters embedded in an amorphous Pd$\mathrm{CeO} 2$ shell (Figure $2 \mathrm{E}, \mathrm{F}$ ), as if this layer would be originated by the dissolution of $\mathrm{Pd}$ nanoclusters into the ceria lattice. A similar situation, with copper clusters dispersed into the ceria lattice at atomic scale, has been recently reported following the mechanical mixing of cerium and copper complexes.[18] This unique embedded configuration might explain the reasons why active Pd4+ centers in PdCeM are more efficient than in PdCelW, where the presence of $\mathrm{Pd} 4+$ (also detected by XPS) is not characterized by the same nanoscale environment. This picture might also explain why promotion of activity is not observed for PdZrM and PdOCeM. Thanks to the oxygen exchange properties of ceria, Pd4+ ions are formed on $\mathrm{CeO} 2$ under conditions much different from other supports.[5b] Moreover, the appearance of these species is prompted by the migration of Pd atoms in the lattice from metallic $\mathrm{Pd}$ clusters on the surface[5b], a situation that likely could not be feasible in presence of stable PdO clusters.

In summary, it is shown that the formation of a specific nanoscale arrangement, with $\mathrm{Pd}$ moieties embedded in the outer surface layers of ceria, promotes methane activation at much lower temperature compared to conventional $\mathrm{Pd} / \mathrm{CeO} 2$ catalysts. These experimental results, supported by a strong theoretical background developed independently[5], open the way to the design of highly active Pd-ceria catalytic systems via sustainable mechanical synthesis routes. In addition, and perhaps more importantly, they put the accent on the role of mechanical stress in modifying at nanoscale the properties of ceria, which can offer a powerful tool in the design of materials with undisclosed properties.

\section{Acknowledgements}

Financial support from Ford Motor Company under 2014-2195R URP Award "Three-way catalyst materials for compressed natural gas vehicles" is kindly acknowledged. MD acknowledges Regione Friuli Venezia Giulia for funding PhD under Operating Program of the European Social Fund 2014/2020. JL is a Serra Hunter Fellow and is grateful to ICREA Academia program and grants MINECO/FEDER ENE2015-63969 and GC 2017 SGR 128. 
[3] a) S. Colussi, A. Trovarelli, C. Cristiani, L. Lietti, G. Groppi, Catalysis Today 2012, 180, 124-130; b) M. Cargnello, J. J. Delgado Jaen, J. C. Hernandez Garrido, K. Bakhmutsky, T. Montini, J. J. Calvino Gamez, R. J. Gorte, P. Fornasiero, Science 2012, 337, 713-717; c) S. Fouladvand, S. Schernich, J. Libuda, H. Gronbeck, T. Pingel, E. Olsson, M. Skoglundh, P. A. Carlsson, Top Catal 2013, 56, 410-415; d) J. Nilsson, P. A. Carlsson, S. Fouladvand, N. M. Martin, J. Gustafson, M. A. Newton, E. Lundgren, H. Gronbeck, M. Skoglundh, ACS Catalysis 2015, 5, 2481-2489.

[4] a) L. Meng, J.-J. Lin, Z.-Y. Pu, L.-F. Luo, A.-P. Jia, W.-X. Huang, M.-F. Luo, J.-Q. Lu, Appl. Catal. B-Environ. 2012, 119, 117-122; b) Y. Zhu, S. R. Zhang, J. J. Shan, L. Nguyen, S. H. Zhan, X. L. Gu, F. Tao, ACS Catalysis 2013, 3, 2627-2639; c) S. Colussi, A. Gayen, M. Boaro, J. Llorca, A. Trovarelli, Chemcatchem 2015, 7, 2222-2229.

[5] a) T. P. Senftle, A. C. T. van Duin, M. J. Janik, ACS Catalysis 2015, 5, 6187-6199; b) T. P. Senftle, A. C. T. van Duin, M. J. Janik, ACS Catalysis 2017, 7, 327-332.

[6] S. Colussi, A. Gayen, M. F. Camellone, M. Boaro, J. Llorca, S. Fabris, A. Trovarelli, Angewandte Chemie-International Edition 2009, 48, 8481-8484.

[7] E. Aneggi, V. Rico-Perez, C. de Leitenburg, S. Maschio, L. Soler, J. Llorca, A. Trovarelli, Angewandte Chemie-International Edition 2015, 54, 14040-14043.

[8] R. Gholami, M. Alyani, K. J. Smith, Catalysts 2015, 5, 561-594.

[9] N. Kamiuchi, H. Muroyama, T. Matsui, R. Kikuchi, K. Eguchi, Appl Catal A-Gen 2010, 379, 148-154.

[10] M. H. Enayati, F. A. Mohamed, Int Mater Rev 2014, 59, 394-416.

[11] R. B. Schwarz, C. C. Koch, Appl Phys Lett 1986, 49, 146-148.

[12] a) Z. Yang, L. A. Zhansheng, G. Luo, K. Hermansson, Phys Lett A 2007, 369, 132-139; b) B. Liu, J. Liu, T. Li, Z. Zhao, X. Q. Gong, Y. Chen, A. J. Duan, G. Y. Jiang, Y. C. Wei, J Phys Chem C 2015, 119, 12923-12934; c) E. M. Slavinskaya, T. Y. Kardash, O. A. Stonkus, R. V. Gulyaev, I. N. Lapin, V. A. Svetlichnyi, A. I. Boronin, Catal Sci Technol 2016, 6, 6650-6666.

[13] a) Y. Maeda, T. Akita, M. Kohyama, Catal Lett 2014, 144, 2086-2090; b) Y. Yang, S. Z. Zhang, S. W. Wang, K. L. Zhang, H. Z. Wang, J. Huang, S. B. Deng, B. Wang, Y. J. Wang, G. Yu, Environ Sci Technol 2015, 49, 4473-4480; c) C. Borchers, M. L. Martin, G. A. Vorobjeva, O. S. Morozova, A. A. Firsova, A. V. Leonov, E. Z. Kurmaev, A. I. Kukharenko, I. S. Zhidkov, S. O. Cholakh, J Nanopart Res 2016, 18; d) C. J. Tang, B. W. Sun, J. F. Sun, X. Hong, Y. Deng, F. Gao, L. Dong, Catalysis Today 2017, 281, 575-582.

[14] a) T. X. T. Sayle, M. Cantoni, U. M. Bhatta, S. C. Parker, S. R. Hall, G. Mobus, M. Molinari, D. Reid, S. Seal, D. C. Sayle, Chemistry of Materials 2012, 24, 1811-1821; b) C. Munnings, S. P. S. Badwal, D. Fini, lonics 2014, 20, 1117-1126.

[15] a) J. E. Spanier, R. D. Robinson, F. Zheng, S. W. Chan, I. P. Herman, Phys Rev B 2001, 64; b) A. Filtschew, K. Hofmann, C. Hess, J Phys Chem C 2016, 120, 6694-6703. 
[16] a) S. Maschio, O. Sbaizero, S. Meriani, Journal of the European Ceramic Society 1992, 9, 127-132; b) G. V. Samsonov, in Handbook of the Physicochemical Properties of the Elements (Ed.: G. V. Samsonov), Springer US, Boston, MA, 1968, pp. 387-446.

[17] H. Krupp, Advances in Colloid and Interface Science 1967, 1, 111-239.

[18] W. C. Zhan, S. Z. Yang, P. F. Zhang, Y. L. Guo, G. Z. Lu, M. F. Chisholm, S. Dai, Chemistry of Materials 2017, 29, 7323-7329. 